Composition and luminescence studies of InGaN epilayers grown at different hydrogen flow rates

This article has been downloaded from IOPscience. Please scroll down to see the full text article.

2013 Semicond. Sci. Technol. 28065011

(http://iopscience.iop.org/0268-1242/28/6/065011)

View the table of contents for this issue, or go to the journal homepage for more

Download details:

IP Address: 130.159.24.44

The article was downloaded on 20/05/2013 at $14: 23$

Please note that terms and conditions apply. 


\title{
Composition and luminescence studies of InGaN epilayers grown at different hydrogen flow rates
}

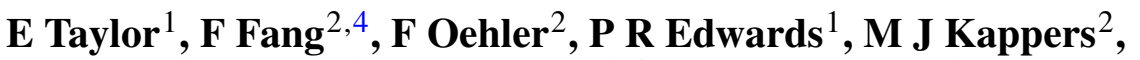 \\ K Lorenz $^{3}$, E Alves ${ }^{3}$, C McAleese ${ }^{2,5}$, C J Humphreys ${ }^{2}$ and R W Martin ${ }^{1}$ \\ ${ }^{1}$ Department of Physics, SUPA, University of Strathclyde, Glasgow G4 0NG, UK \\ ${ }^{2}$ Department of Materials Science and Metallurgy, University of Cambridge, Cambridge CB2 3QZ, UK \\ ${ }^{3}$ IST/ITN, Instituto Superior Técnico, 2686-953 Sacavém, Portugal \\ E-mail: elaine.taylor@strath.ac.uk
}

Received 10 December 2012, in final form 12 March 2013

Published 16 May 2013

Online at stacks.iop.org/SST/28/065011

\begin{abstract}
Indium gallium nitride $\left(\operatorname{In}_{x} \mathrm{Ga}_{1-x} \mathrm{~N}\right)$ is a technologically important material for many optoelectronic devices, including LEDs and solar cells, but it remains a challenge to incorporate high levels of InN into the alloy while maintaining sample quality. A series of InGaN epilayers was grown with different hydrogen flow rates $(0-200 \mathrm{sccm})$ and growth temperatures $\left(680-750^{\circ} \mathrm{C}\right)$ to obtain various InN fractions and bright emission in the range 390-480 nm. These 160-nm thick epilayers were characterized through several compositional techniques (wavelength dispersive $\mathrm{x}$-ray spectroscopy, $\mathrm{x}$-ray diffraction, Rutherford backscattering spectrometry) and cathodoluminescence hyperspectral imaging. The compositional analysis with the different techniques shows good agreement when taking into account compositional gradients evidenced in these layers. The addition of small amounts of hydrogen to the gas flow at lower growth temperatures is shown to maintain a high surface quality and luminescence homogeneity. This allowed InN fractions of up to $\sim 16 \%$ to be incorporated with minimal peak energy variations over a mapped area while keeping a high material quality.
\end{abstract}

(Some figures may appear in colour only in the online journal)

\section{Introduction}

Research on III-nitride materials for use in optoelectronic devices has advanced in recent years, with the emergence of high efficiency blue light-emitting diodes (LEDs) and laser diodes, but achieving highly efficient nitride-based green LEDs still poses problems. The most common alloy used for achieving green emission is InGaN, but the drop in efficiency with increasing $\mathrm{InN}$ fraction is marked and still not fully understood. It is well known that the reduction in growth temperature allows the increase of the $\mathrm{InN}$ fraction within

\footnotetext{
${ }^{4}$ Present address: Quantum Wafer Inc., Building A, Golden Valley, Pingzhou, Guangzhou, People's Republic of China.

${ }^{5}$ Present address: Aixtron Ltd., Buckingway Business Park, Anderson Road, Swavesey, Cambridge CB24 4FQ, UK
}

the alloy, shifting the wavelength into the green [1], but the ammonia used to provide nitrogen during growth is difficult to dissociate at these lower temperatures, resulting in a reduced InGaN layer quality. Incorporating large $\mathrm{InN}$ fractions can also lead to strain-induced phase separation producing layers with spatially inhomogeneous luminescence [2]. The challenge still remains to obtain high $\mathrm{InN}$ fractions within $\mathrm{InGaN}$ alloys while maintaining sample quality and luminescence homogeneity, especially for relatively thick layers $(>50 \mathrm{~nm}$ ). The addition of small amounts of hydrogen carrier gas during growth has been seen to improve the sample quality but at the expense of a reduction in the $\mathrm{InN}$ incorporation [3-6].

In this work we characterized a set of $160-\mathrm{nm}$ thick InGaN epilayers to determine the effect of adding small amounts of hydrogen to the gas flow, during growth at lower temperatures, on the composition and luminescence 
properties of the material. The composition was measured using wavelength dispersive $\mathrm{x}$-ray (WDX) spectroscopy, $\mathrm{x}$-ray diffraction (XRD) and Rutherford backscattering spectrometry (RBS). The luminescence properties were explored using cathodoluminescence (CL) hyperspectral imaging, in which the sample is rastered under an electron beam using a precision scanning stage and the emission spectrum recorded at each point to build up a multidimensional luminescence dataset $[7,8]$.

\section{Experimental details}

A series of InGaN epilayer samples was grown on c-plane GaN/sapphire substrates using a Thomas Swan $6 \times$ 2-inch close-coupled showerhead metalorganic vapour phase epitaxy reactor using trimethylindium (TMI), trimethylgallium (TMG) and ammonia $\left(\mathrm{NH}_{3}\right)$ as source materials. Details of the GaN epilayer growth can be found elsewhere [9]. The threading dislocation density of the GaN template was about $5 \times 10^{8} \mathrm{~cm}^{-2}$ as measured by counting the pit density in atomic force microscopy (AFM) images after the sample surface was subjected to treatment with silane and ammonia [10]. For the InGaN deposition the main carrier gas used was nitrogen with small quantities of hydrogen (up to 200 $\mathrm{sccm}$ (standard cubic centimeters)) added for certain layers, while using TMI flow of $23.0 \mu \mathrm{mol} \mathrm{min}{ }^{-1}$, a TMG flow of $11.2 \mu \mathrm{mol} \mathrm{min}{ }^{-1}$ and an ammonia flow of $446 \mathrm{mmol} \mathrm{min}^{-1}$ at a reactor pressure of 300 Torr. The samples were grown with an InGaN layer of nominal thickness $160 \mathrm{~nm}$ and with target InN fractions varying from $5 \%$ to $20 \%$, which was achieved by varying growth temperature and hydrogen flow rate, as shown in table 1. The temperatures quoted in this paper are the susceptor temperature measured using a pyrometer calibrated against a black body source. Based on the layer thicknesses determined by XRD and RBS, the InGaN growth rate varied between approximately 1.6 and $1.9 \mathrm{~nm} \mathrm{~min} \mathrm{~m}^{-1}$ depending on the alloy composition. The WDX composition and CL measurements were carried out on a modified Cameca SX100 electron probe micro-analyser (EPMA) with an attached optical spectrograph, and the XRD data was measured using a Philips X'Pert MRD diffractometer. The RBS measurements were performed using 1.5 and $2 \mathrm{MeV}$ $\mathrm{He}^{+}$ions and both spectra were fitted simultaneously to a two-layer model using the RBS analysis code NDF [11]. AFM measurements were performed in tapping mode using a Digital Instruments Nanoscope Dimension 3100 microscope, and the data were analysed using the WSXM software package [12].

\section{Results and discussion}

WDX spectroscopy was used to obtain the InN composition of each InGaN epilayer sample. We compare this with the XRD and RBS data as shown figure 1. Figure 1 displays the known trend of decreasing InN fraction with either increasing growth temperature (labelled D to A) or increasing hydrogen flow rate (labelled 00 to 200 after the equivalent flow rate in sccm). All three techniques are in agreement with this trend, but the InN fractions measured using XRD are consistently lower than

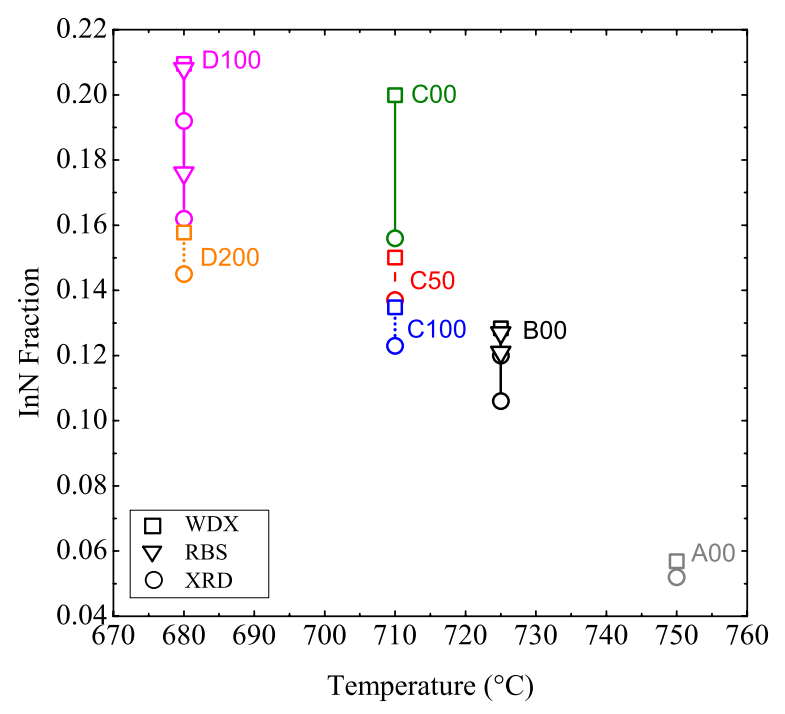

Figure 1. Comparison of average $\mathrm{InN}$ fraction for each of the InGaN samples grown at different growth temperatures and hydrogen flow rates, measured using WDX and XRD. Additionally, RBS data are shown for B00 and D100. Each technique is defined by a different symbol, and each sample is joined by a different line style. For B00 and D100 the extremes of linear fits to the XRD and RBS data are shown to highlight the compositional gradient through the InGaN layer.

those measured using WDX. This difference is attributed to a compositional gradient through the layer, with the higher InN fraction lying close to the surface. The excitation volume of the $5 \mathrm{keV}$ electron beam within the InGaN layer is less than the XRD sampling volume, with XRD providing more of a bulk measurement [13]; a higher $\mathrm{InN}$ fraction nearer the surface will thus disproportionately influence the WDX technique. RBS measurements were performed on two of the samples to test this hypothesis by providing depth-profile information. The RBS spectra for samples B00 and D100 were each fitted using a two layer model, in which the thickness and composition of two uniform layers was varied. The spectra and fits for sample D100 are shown in figure 2(a).

The spectra clearly show a reduction in indium counts for both epilayers as the energy reduces, with this negative slope more clearly visible in the magnified portion of the D100 trace shown in figure $2(b)$. This confirms that there is a variation of $\mathrm{InN}$ content with depth within the $\mathrm{InGaN}$ layer, with the higher $\mathrm{InN}$ composition near the surface. The composition gradients are estimated by fitting the RBS data using the approximation of two layers of different $\mathrm{InN}$ fractions, as reported in table 1 . Using this RBS result as a guide the XRD measurements were further analysed on samples B00 and D100. Reciprocal space maps, as shown in figure 3, revealed broad InGaN peaks relative to the $\mathrm{GaN}$ template peaks. The $\mathrm{GaN}$ templates are under slight in-plane compressive strain, $\left(-1.6\right.$ and $-1.7 \times 10^{-3}$ for B00 and D100, respectively) due to mismatches in thermal expansion during cool down along with differences in $\mathrm{GaN}$ template thickness. These strain values match the RSM data in figure 3 after the necessary rotation correction to account for small differences in the mounting of the samples. The InGaN peak broadening is greater for sample D100 with the higher average $\mathrm{InN}$ fraction 

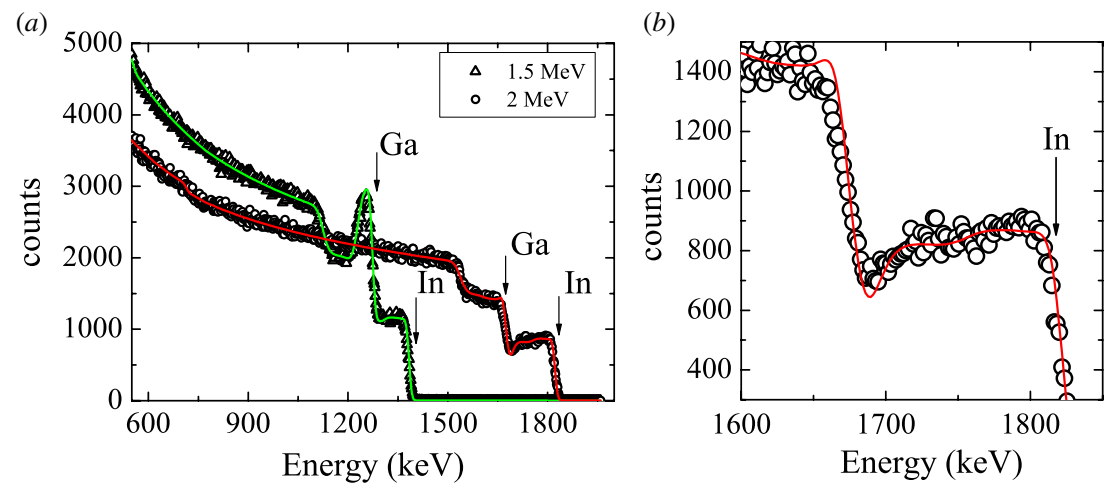

Figure 2. (a) RBS spectra of sample D100 measured with 1.5 and $2 \mathrm{MeV} \mathrm{He}^{+}$ions and using a two layer model for the fit, indicating an indium compositional change through the InGaN layer. (b) Close up of the $2 \mathrm{MeV}$ spectrum at the indium signal to highlight the gradient and two-layer fit.

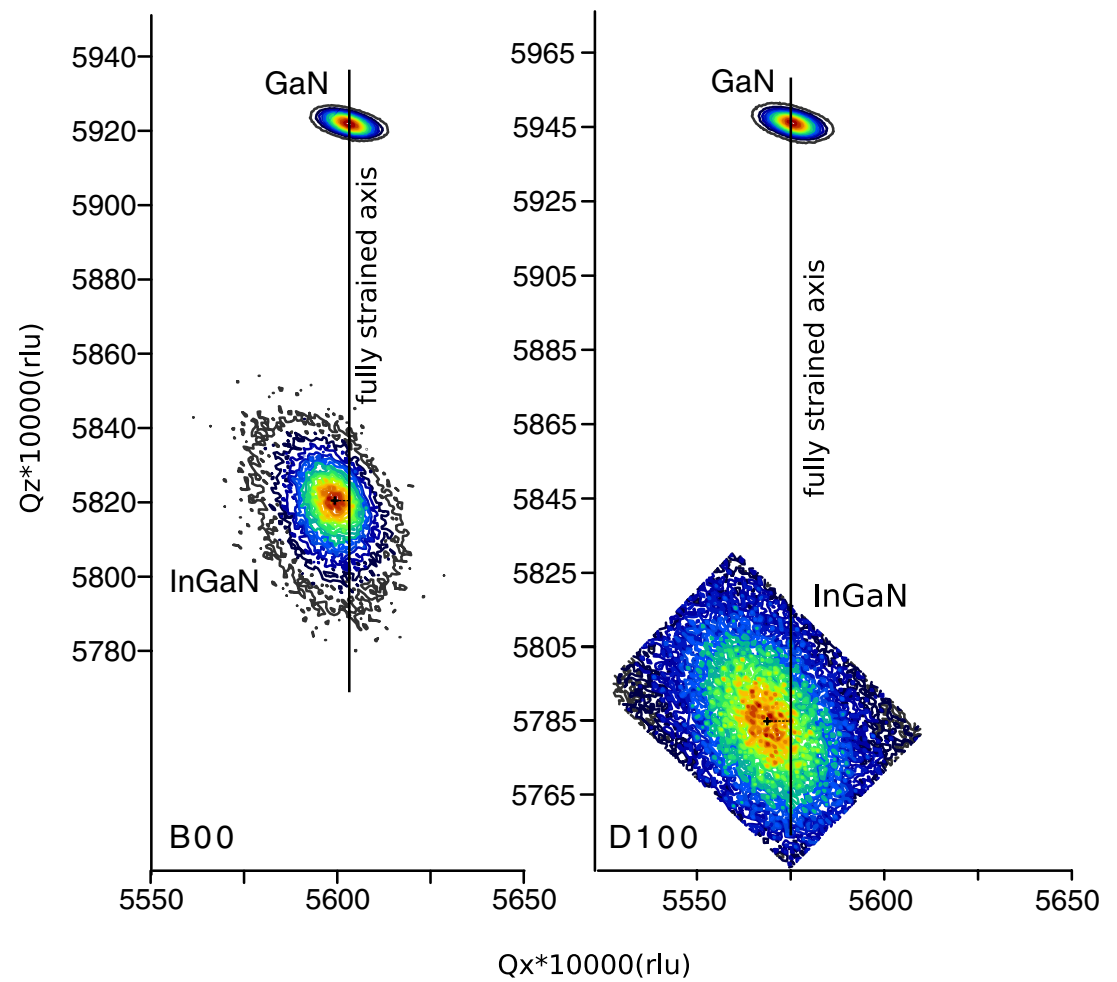

Figure 3. Reciprocal space maps around the 204 reflection of samples B00 (left) and D100 (right).

Table 1. Growth temperature, hydrogen flow rate and InN composition of the samples under study, measured using WDX, XRD and RBS techniques. Values shown for XRD* and RBS* are the extremes of a linear compositional gradient, using the RBS two-layer model as a guide (see text).

\begin{tabular}{lllllllll}
\hline & A00 & B00 & C00 & C50 & C100 & D00 & D100 & D200 \\
\hline Growth temperature $\left({ }^{\circ} \mathrm{C}\right)$ & 750 & 725 & 710 & 710 & 710 & 680 & 680 & 680 \\
Hydrogen flow rate $(\mathrm{sccm})$ & 0 & 0 & 0 & 50 & 100 & 0 & 100 & 200 \\
WDX composition \% & 5.7 & 12.8 & 20.0 & 15.0 & 13.5 & 31.4 & 21.0 & 15.8 \\
XRD average composition \% & 5.2 & 11.5 & 15.6 & 13.7 & 12.3 & 21.0 & 17.8 & 14.5 \\
RBS composition \% (two layer model) & & $12.2-12.5$ & & & & & $18.4-20.0$ & \\
XRD* composition \% (linear model) & & $10.8-12.2$ & & & & & $16.2-19.2$ & \\
RBS* composition \% (linear model) & & $12.1-12.7$ & & & & & $17.6-20.8$ & \\
\hline
\end{tabular}

suggesting a reduced crystal quality. For both samples, the small horizontal shift of the InGaN peak with respect to the $\mathrm{GaN}$ peak is indicative of a partial in-plane relaxation, $2 \%$ for sample B00 and $7 \%$ for sample D100, but no clear evidence was found of a relaxation gradient through the film thickness $[14,15]$. Thus, the XRD model consisted of a single InGaN layer with a constant relaxation value but a linear variation of the InN fraction through the thickness of the film. 


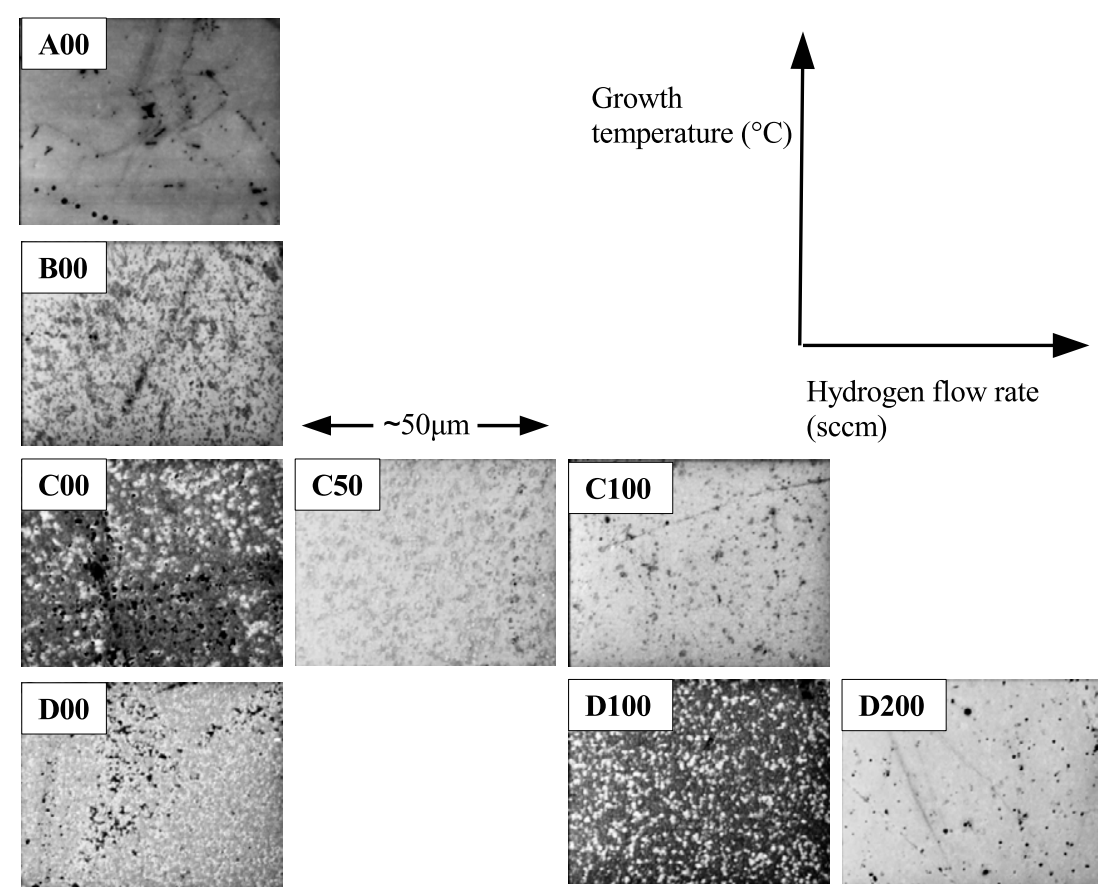

Figure 4. Secondary electron images showing variations in surface quality with change in growth temperature and hydrogen flow rate, indicated by the key in the top right of the figure.

The XRD models found an InN gradient covering a change of 1.4 at. \% (from 10.8 to $12.2 \%$ ) with a partial relaxation of $2 \%$ for sample B00, and an InN gradient covering a change of 3.0 at.\% (from 16.2 to $19.2 \%$ ) with a partial relaxation of $7 \%$ for sample D100. In order to make comparison between the XRD and the RBS data, the two-layer model used to interpret the RBS data was transformed into the equivalent linear variation with the weighted average InN fraction as shown in table 1. The average InN fractions determined by XRD are consistently lower than the RBS values: $11.5 \%$ versus $12.4 \%$ for sample B00 and $17.7 \%$ versus $19.2 \%$ for sample D100, respectively, while the change in InN fraction measured by XRD was found to be greater than the calculated RBS linear change for sample B00 (1.4\% compared with $0.6 \%$ ) but the results were comparable for sample D100 (3.0\% compared with 3.2\%). The upper and lower InN fractions which correspond to these gradients for both XRD and RBS are presented in figure 1 and table 1 . The uncertainties associated with each of the data sets are all approximately $\pm 1 \%$ (absolute in $x$ ) or less.

In addition to the analysis of the composition as a function of depth we performed spatial studies to gain information on surface quality and luminescence homogeneity. Secondary electron (SE) images were taken using the EPMA to look at the differences in surface quality over the sample set. These SE images are shown in figure 4, and illustrate that the surface quality worsens with decreasing growth temperature but improves with increasing hydrogen flow rate for samples grown at the same temperature. These images are compared with the results of AFM as shown in figure 5 .

The AFM images illustrate the morphological evolution with temperature and added hydrogen flow rate used for the InGaN epilayer growth. The InGaN samples A00 and B00 show smooth surface morphology in a standard $\mathrm{H}_{2}$ free environment (figures 5(a) and (b), resp.). It should be noted that the black surface pits are V-defects and their density at approximately $4 \times 10^{8} \mathrm{~cm}^{-2}$ in sample A00 corresponds to the threading defect density in the GaN template underneath. The additional pits seen in surface of B00 that are arranged in a circular fashion, may originate from inversion domains bounded by stacking mismatch boundaries formed at the $\mathrm{GaN} / \mathrm{InGaN}$ interface $[16,17]$. The surface morphology of the InGaN epilayers becomes worse when the indium content is increased by reducing the growth temperature; figure 5(c) of sample $\mathrm{C} 00$ shows additional island formation while the surface of D00 in figure $5(d)$ is covered with small round islands and mounds. Adding hydrogen during the $\mathrm{InGaN}$ growth at the same growth temperature improves the surface significantly, e.g. compare $\mathrm{C} 100$ and $\mathrm{C} 50$ with $\mathrm{C} 00$ or D100 and D50 with D00; the large clusters of islands change with added hydrogen flow to far fewer and smaller inclusions on the surface. The transition from a poor morphology of samples C00, D00 and D100 to the much improved surface quality of samples C100 and D200 is also recognizable from the SEM images in figure 4.

The better quality samples (A00, B00, C100 and D200) were further investigated by taking higher resolution CL hyperspectral images to see the relationship between luminescence homogeneity, growth temperature and hydrogen flow rate. The CL images shown in figure 6(a) were generated by fitting a Gaussian curve to the InGaN emission peak of each spectrum in the hyperspectral image, and plotting the resultant peak energies as a function of position. An example of a fitted spectrum, taken from map B00, is shown in figure 6(b). A visual comparison of luminescence homogeneity was accomplished by setting the range of the scale bars of each map to be the same, with the centre of the scale set to the mean 
(a)

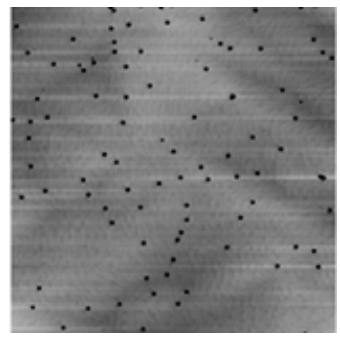

A00

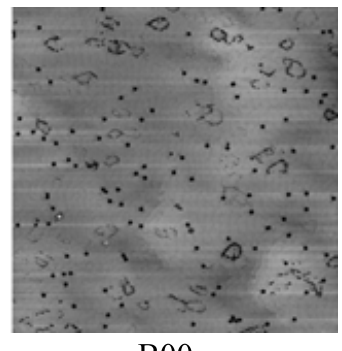

$\mathrm{B} 00$ (c)

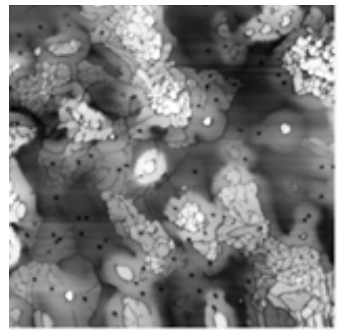

$\mathrm{CO0}$

(d)

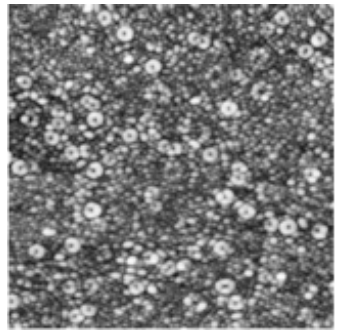

D00

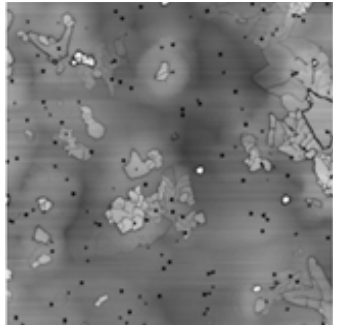

$\mathrm{C} 50$

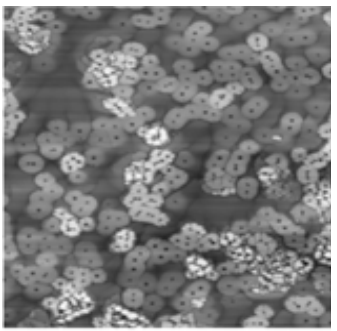

D100

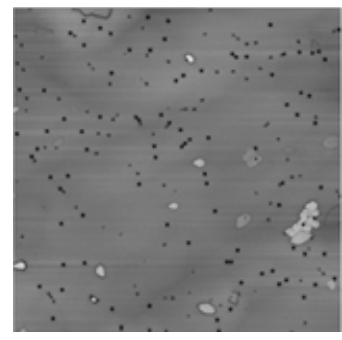

$\mathrm{C} 100$

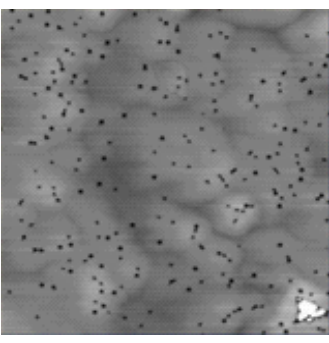

D200

Figure 5. AFM images of InGaN epilayers $(5 \mu \mathrm{m} \times 5 \mu \mathrm{m})$ from series A, B, C and D. Image height scales are $50 \mathrm{~nm}$ for A00 in $(a)$ and B00 in $(b), 80 \mathrm{~nm}$ for all images in $(c)$ and D200 in $(d)$ and $250 \mathrm{~nm}$ for D00 and D100 in $(d)$.

(a)
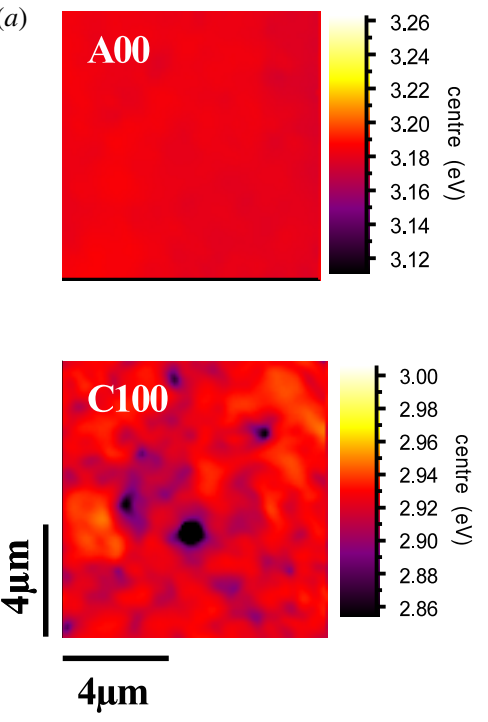

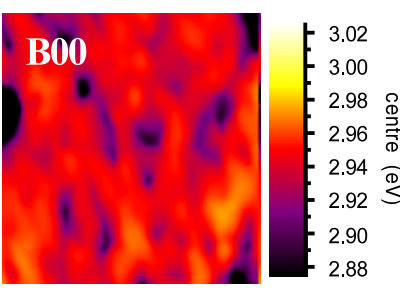

(b)
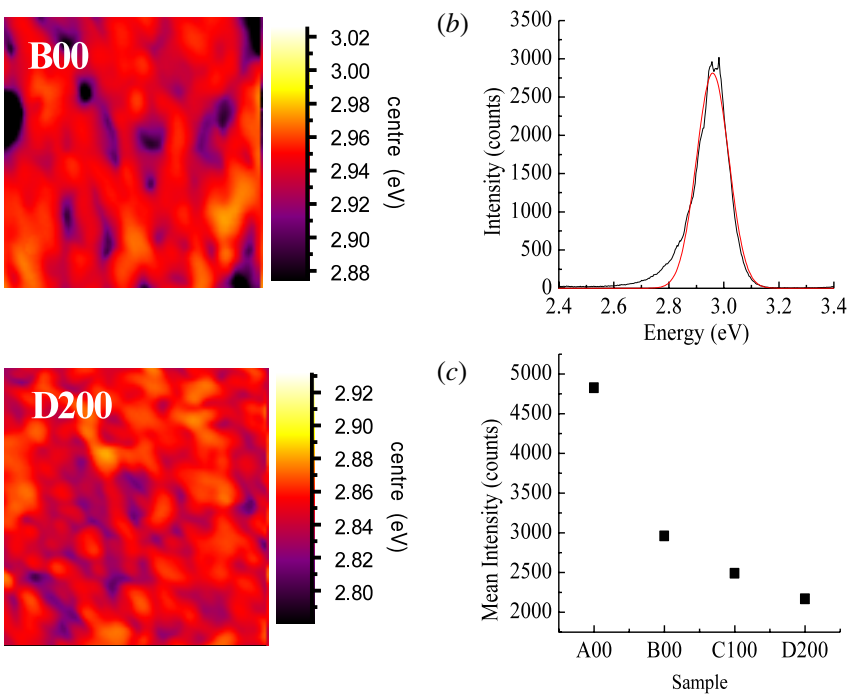

Figure 6. (a) Subsets of the CL hyperspectral images showing the change in peak energy centre over the mapped area of each sample. The scales of each map are centred on the mean peak energy of each sample and the range is kept constant across all maps allowing a comparison to be made. (b) A typical spectrum taken, with fit, from map B00 and (c) average peak CL intensity of each of the maps shown.

peak energy of each individual map. In addition the mean maximum intensity of the CL peak of each map are shown in figure $6(c)$, allowing a comparison of quantum efficiency to be made. The figure shows an overall decrease in mean CL intensity with decreasing growth temperature, but by a reduced rate for the samples grown with the addition of hydrogen.

The CL mapping illustrates an increase in peak centre variation with decreasing growth temperature, but this effect 


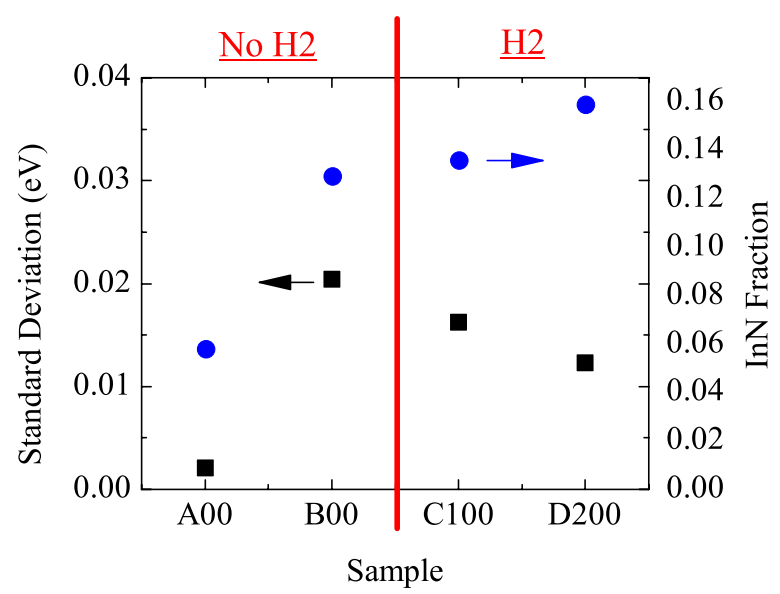

Figure 7. Quantified energy variation and average $\mathrm{InN}$ fraction for the CL maps (as shown in figure 3) using the standard deviation of the peak energy variation.

is reduced with the addition of hydrogen; for example the map of sample D200 $\left(680{ }^{\circ} \mathrm{C}, 200 \mathrm{sccm}, 15.8 \% \mathrm{InN}\right)$ is seen to have less variation in peak centre across the map than sample B00 $\left(725{ }^{\circ} \mathrm{C}, 0 \mathrm{sccm}, 12.8 \%\right)$ despite its high $\mathrm{InN}$ fraction. In order to compare the luminescence homogeneity and quality, the variation of peak position in each map was quantified, by calculating the standard deviation over the entire map. The standard deviation highlights the energy spread within the measured area and is compared with the WDX composition for the different samples in figure 7. The standard deviation in the peak energy is seen to reduce on going from samples B00 to C100 to D200 (i.e. on decreasing the growth temperature and increasing the hydrogen flow rate). This further emphasizes that introducing small amounts of hydrogen into the carrier gas during growth at lower temperatures improves the luminescence homogeneity. The data further highlight that both a higher InN fraction and better luminescence homogeneity can simultaneously be achieved by introducing a small hydrogen flow into the growth procedure.

\section{Conclusions}

The composition and luminescence properties of a set of InGaN epilayers grown with varying temperature and hydrogen flow rate have been analysed. Compositional data from various techniques revealed the presence of an indium gradient through the layer for which the higher composition was found to lie near the surface of the layer. The gradient amplitude increased with increasing average $\mathrm{InN}$ fraction. The addition of small flows of hydrogen to the nitrogen carrier gas during the InGaN deposition drastically reduced the $\mathrm{InN}$ fraction. The loss of indium content could be compensated by lowering the InGaN growth temperature. Despite this lower temperature, the samples grown with small amounts of hydrogen were seen to improve in surface quality. The increase in hydrogen flow rate also had a positive effect on the luminescence homogeneity with the cathodoluminescence maps of the higher quality samples showing a reduction in peak energy variation. By quantifying these emission energy variations we have shown that $160 \mathrm{~nm}$-thick InGaN samples with an average $\mathrm{InN}$ fraction of $10-15 \%$ and grown with a small hydrogen flow have a reduced spatial inhomogeneity of the emission energy compared to equivalent samples grown without hydrogen.

\section{Acknowledgments}

This work is part of the Lighting the Future project and was supported through UK EPSRC grant EP/I012591/1.

\section{References}

[1] Chang P C, Yu C L, Jahn Y W, Chang S J and Lee K H 2011 Effect of growth temperature on the indium incorporation in InGaN epitaxial films Adv. Mater. Res. 287 1456-9

[2] Tamulaitis G 2011 Spatial inhomogeneity of luminescence in III-nitride compounds Mater. Sci. 17 343-51

[3] Sadler T C, Kappers M J and Oliver R A 2011 The impact of hydrogen on indium incorporation and surface accumulation in InAlN epitaxy J. Cryst. Growth 331 4-7

[4] Bosi M and Fornari R 2004 A study of indium incorporation efficiency in InGaN grown by MOVPE J. Cryst. Growth 265 434-9

[5] Piner E L, Behbehani M K, El-Masry N A, McIntosh F G, Roberts J C, Boutros K S and Bedair S M 1997 Effect of hydrogen on the indium incorporation in InGaN epitaxial films Appl. Phys. Lett. 70 461-3

[6] Bosi M, Fornari R, Scardova S, Avella M, Martínez O and Jimenez J 2004 Compositional and optical uniformity of InGaN layers deposited on (0001) sapphire by metalorganic vapour phase epitaxy Semicond. Sci. Technol. 19 147-51

[7] Edwards P R and Martin R W 2011 Cathodoluminescence nano-characterization of semiconductors Semicond. Sci. Technol. 26064005

[8] Martin R W, Edwards P R, O’Donnell K P, Dawson M D, Jeon C-W, Liu C, Rice G R and Watson I M 2004 Cathodoluminescence spectral mapping of III-nitride structures Phys. Status Solidi a 201 665-72

[9] Kappers M J, Datta R, Oliver R A, Rayment F D G, Vickers M E and Humphreys C J 2007 Threading dislocation reduction in (0001) GaN thin films using $\mathrm{SiN}_{x}$ interlayers J. Cryst. Growth 300 70-4

[10] Oliver R A, Kappers M J, Sumner J, Datta R and Humphreys C J 2006 Highlighting threading dislocations in MOVPE-grown GaN using an in situ treatment with $\mathrm{SiH}_{4}$ and $\mathrm{NH}_{3}$ J. Cryst. Growth 289 506-14

[11] Barradas N P, Jeynes C and Webb R P 1997 Simulated annealing analysis of Rutherford backscattering data Appl. Phys. Lett. 71 291-3

[12] Horcas I, Fernández R, Gómez-Rodríguez J M, Colchero J, Gómez-Herrero J and Baro A M 2007 WSXM: a software for scanning probe microscopy and a tool for nanotechnology Rev. Sci. Instrum. 78013705

[13] Pereira S, Correia M R, Pereira E, O’Donnell K P, Trager-Cowan C, Sweeney F and Alves E 2001 Compositional pulling effects in $\mathrm{In}_{x} \mathrm{Ga}_{1-x} \mathrm{~N} / \mathrm{GaN}$ layers: a combined depth-resolved cathodoluminescence and Rutherford backscattering/channeling study Phys. Rev. B 64205311

[14] Pereira S, Correia M R, Pereira E, O'Donnell K P, Alves E, Sequeira A D, Franco N, Watson I M and Deatcher C J 2002 Strain and composition distributions in wurtzite InGaN/GaN layers extracted from x-ray reciprocal space mapping Appl. Phys. Lett. 80 3913-5 
[15] Pereira S, O’Donnell K P and Alves E 2007 Role of nanoscale strain inhomogeneity on the light emission from $\mathrm{InGaN}$ epilayers Adv. Funct. Mater. 17 37-42

[16] Massabuau F C-P, Sahonta S-L, Trinh-Xuan L, Rhode S, Puchtler T J, Kappers M J, Humphreys C J and Oliver R A 2012 Morphological, structural, and emission characterization of trench defects in $\mathrm{InGaN} / \mathrm{GaN}$ quantum well structures Appl. Phys. Lett. 101212107

[17] Bruckbauer J, Edwards P R, Wang T and Martin R W 2011

High resolution cathodoluminescence hyperspectral imaging of surface features in InGaN/GaN multiple quantum well structures Appl. Phys. Lett. 98141908 